\title{
CREACIÓN DE UN HÉROE EN INGLÉS PARA EDUCANDO HOSPITALARIO: UN ESTUDIO DE CASO
}

\section{HERO CREATION IN ENGLISH FOR AN EDUCANDO HOSPITALARIO: A CASE STUDY}

\section{Gersson Joaquín Buitrago García ${ }^{1}$}

\section{Juan Camilo Villalobos Quintero²}

\section{Astrid Ramirez Valencia ${ }^{3}$}

Universidad Distrital Francisco José de Caldas

Bogotá, Colombia

\section{RESUMEN}

El contexto educativo depende de las necesidades de los estudiantes, los educandos hospitalarios por su parte demandan una pedagogía enfocada hacia su situación, en la cual convergen factores tales como; médico, familiar, social y académico. Teniendo en cuenta estrategias pertinentes para la participación de 1 Estudiante de pregrado de la licenciatura en educación básica con énfasis en

inglés, Universidad Distrital Francisco José de Caldas, Docente de inglés en

diferentes centros de idiomas. CORREO: gjbuitragog@ correo.udistrital.edu.co

ORCID: https://orcid.org/0000-0002-7562-1026

2 Estudiante de pregrado de la licenciatura en educación básica con énfasis en

inglés, Universidad Distrital Francisco José de Caldas, Docente de inglés en

diferentes centros de idiomas. CORREO: jcvillalobosq@ correo.udistrital.edu.co

ORCID: https://orcid.org/0000-0003-0534-4550

3 Docente Universidad Distrital Francisco José de Caldas Bogotá Colombia, Ph.D. en Lenguaje y cultura, formadora de profesores de inglés durante más de 25 años. Investigadora y maestra en la Universidad Distrital Francisco José de Caldas. CORREO:aramirezv@udistrital.edu.co ORCID: https://orcid.org/0000-0002-3025-598 procesos educativos, El objetivo de este estudio de caso es el análisis del proceso de arteterapia estrategia implementada en un educando hospitalario quien por medio de la creación del héroe en inglés y su experiencia hace un manejo del duelo.

Para el grupo de sesiones se consideran diferentes teorías, Kolb (2015), Romero (2014), Klein (2006), entre otros, donde es el enfoque del aprendizaje experiencial el protagonista de las sesiones la cual facilita el proceso de creación por medio del inglés además del manejo del duelo. Los instrumentos que se aplicaron en esta investigación fueron: entrevistas, notas post-facto, artefactos de los estudiantes. Con el objetivo de abordar el proceso de duelo y el educando hospitalario en su condición desde la perspectiva pedagógica de la enseñanza del inglés y. Por último, la interpretación da cuenta de resultados donde los factores socio-académicos 
y situaciones fluctuantes son determinantes a lo largo de la investigación.

\section{PALABRAS CLAVE}

Educando hospitalario, manejo del duelo, arteterapia, creación del héroe, inglés como segunda lengua.

\section{ABSTRACT}

The educational context depends on the needs of the students; hospital students demand pedagogy focused on their situation, in which factors such as medical, family, social and academic converge. Taking into account relevant strategies for the participation of educational processes. The objective of this case study is the analysis of a hospitalized student who through art therapy, and by means of the creation of the hero and his experience, manages grief.

For the group of sessions different theories are considered, Kolb (2015), Romero (2014), Klein (2006), among others, where the experience is the protagonist of the sessions which facilitates the process of creation through English learning in addition to grief management. The instruments applied in this research were: interviews, postfacto notes, student artifacts. With the objective of understanding the hospitalized learner and the process of mourning for his condition. Finally, the part of the interpretation manages to evidence important socio-academic factors in addition to fluctuating situations presented in the research.

\section{KEY WORDS}

Educando hospitalario, grief management, art therapy, creation of the hero, English as a second language.

\section{INTRODUCCIÓN}

Las tendencias de educación actual llevan a los docentes a encontrar no solamente nuevas estrategias de enseñanza sino también nuevos ambientes, estudiantes y lugares donde la educación se ha hecho necesaria desde hace un tiempo pero que hasta la actualidad parece estar llegando. Teniendo en cuenta lo anterior, el concepto de inclusión juega un papel vital ya que existen lugares y personas los cuales no están inmersos en los procesos educativos, de allí que los profesores se vean enfrentados a nuevos retos dentro de la inclusión, la cual a su vez está sujeta tanto a las necesidades de los estudiantes como en el contexto donde esto ocurre.

Apoyando la idea anterior, Blanco (2006) afirma que la inclusión pasa necesariamente por diferentes niveles de la sociedad, aquí nos centramos en uno de esos, la educación, donde es obligatorio crear espacios diferentes a los que ya están establecidos. (p.22) La inclusión en la educación actual puede explorar diversos campos como el aula hospitalaria, la inclusión social además del aula regular.

Tradicionalmente, la inclusión se da dentro de los entornos escolares atendiendo las necesidades especiales de la población presente, pero este proceso se ve obstaculizado cuando precisamente, por su necesidad, el alumno no puede asistir a la escuela. Es aquí cuando el estudiante pierde el derecho a recibir una educación que se adapte a su necesidad o necesidades, por la falta de un sistema educativo que le permita a la persona recibir educación aun estando en condiciones que no le permiten desplazarse al aula. De acuerdo con Steinke et al. (2016) "Niños que han sido hospitalizados con frecuencia o por largos periodos de tiempo experimentan una gran cantidad de situaciones estresantes únicas, incluida la separación de padres, hermanos y compañeros, así como la exposición a numerosos procedimientos médicos dolorosos." (p.02). Es por ello que este trabajo está tomando como participante 
un educando hospitalario quien se encuentra experimentando este proceso y de esta manera está expuesto a sufrir depresión por estar aislado de su contexto habitual (Kubler-Ross 2005). En este caso, la inclusión se debe enfocar en aquel estudiante que tuvo, debido a su condición médica, que abandonar el colegio, es por eso que se plantea un apoyo a través del arte que permitió desarrollar un proceso en el que puede conectar el aprendizaje del inglés, el arte y a la vez se puede disminuir el impacto negativo que un contexto clínico puede llevar consigo para una persona sin importar su edad.

Por un lado, el educando hospitalario está expuesto a diversos sentimientos, como se mencionó anteriormente; el docenteinvestigador asume la responsabilidad de acercar las vivencias de ese contexto para que el educando hospitalario a través de estrategias pedagógicas y acompañado de sus familiares tenga un mejor manejo de esos sentimientos. El recibir estas herramientas para la superación de estas situaciones pueden ayudar al educando hospitalario no solo a sobrellevar distintas emociones sino también a contribuir en el mejoramiento de su estado de salud.

Por otro lado, para llevar a cabo procesos inclusivos efectivos, es obligatorio involucrar a la comunidad, lo cual incluye a los padres, familia, médicos, enfermeras, psicólogos, cuidadores, terapeutas y toda la comunidad escolar, que se compromete a crear un ambiente donde nadie esté sobre las necesidades del otro $y$ trabajan codo a codo con metas colectivas, para superar las dificultades que puedan tener los estudiantes, no solo en la educación sino en la sociedad y la familia.

Además, los maestros tienen el desafío constante de enfrentar nuevos entornos o trabajar dentro de poblaciones diversas, lo que los obliga a cambiar su visión del aula. Para este proyecto, el contexto hospitalario se convirtió en el aula de inglés del hospital con el fin de crear un ambiente donde el educando hospitalario pueda convertir su experiencia en conocimiento a través de la creación de un héroe. El propósito de este estudio de caso cualitativo es analizar el proceso de arteterapia a través de la creación de héroe en clases de inglés por parte del educando hospitalario para el manejo del duelo.

\section{JUSTIFICACIÓN}

Hoy en día, la importancia de la inclusión en la educación pasa por diferentes contextos, necesidades, innovaciones y creatividad para enriquecer los conocimientos de los alumnos, sus experiencias vitales y asimismo desde el campo pedagógico comprometernos con alternativas en el proceso de aprendizaje. "A través de la arteterapia, las personas con discapacidad tienen la oportunidad de poder comunicar sus sentimientos y necesidades a los demás de una manera más perceptible. (Barbero 2007 p.174)". La aplicación de la arteterapia ofrece al educando hospitalario la posibilidad de transmitir su historia a través de la representación de un héroe y su contexto, pudiendo este ser discapacitante en tanto limita al educando hospitalario a continuar su cotidianidad social y regularidad académica. Además, los docentesinvestigadores participantes de este estudio les dan herramientas en inglés para construirlo. Esta estrategia de creación de héroes está orientada a dar a los estudiantes un medio para expresar sus sentimientos y pensamientos.

De igual manera (Carbo, 2016) habla de la importancia de llevar el trabajo artístico terapéutico a aquellos niños que necesitan especialmente mejorar su capacidad de percibir y expresar emociones; esto, con el fin de crear ambientes seguros donde no experimenten ningún contratiempo psicológico, además de 
facilitar su adaptación a nuevos contextos como el aula hospitalaria donde estarán aislados o no podrán asistir a la escuela. Es por ello que este proyecto va dirigido a este tipo de población, teniendo en cuenta sus necesidades y contexto.

\section{IDENTIFICACIÓN DEL PROBLEMA}

En esta sección se plantea el problema que se evidencia con respecto al educando hospitalario y su proceso educativo. Desde la descripción del panorama general de la situación a tratar y los conceptos pertinentes, es por ello que se resalta la importancia de la inclusión en procesos educativos, ya que esta debe ser dirigida por diferentes contextos, necesidades, innovaciones además de la creatividad con el objetivo de enriquecer el conocimiento de los estudiantes, teniendo en cuenta sus experiencias de vida para su proceso educativo.

La UNESCO (2015) proporciona la definición de inclusión la cual es pertinente en este estudio, esta es definida como: "el proceso de identificar y responder a diversas necesidades de todos los estudiantes a través de su participación en el aprendizaje, cultura, y comunidad, reduciendo cualquier exclusión en educación". Como es explicado, la inclusión necesita cubrir todas las necesidades y los lugares en términos educativos, a ello se refiere que es necesario realizar procesos y estrategias inclusivas en cualquier contexto educativo. Teniendo en cuenta este punto de vista, profesores $y$ escuelas juegan un rol importante para desarrollar actividades inclusivas, pero cuando el entorno de aprendizaje se encuentra fuera del colegio se requieren de estrategias para hacer que el educando hospitalario tenga un espacio académico en el cual logre desenvolverse sin dificultades o con las mínimas posibles.

Estudios existentes muestran diferentes intervenciones donde se trabaja con educandos hospitalarios, estos estudios están basados en metodologías desarrolladas donde se incluye la arteterapia, la cual consiste en un acompañamiento a través de expresiones artísticas (Klein, 2006, p.13). Esta metodología permite al educando hospitalario crear su historia a través de la representación de un héroe y su contexto para el manejo del duelo. Esta estrategia está orientada para dar al educando hospitalario la oportunidad de expresar sus sentimientos y pensamientos hacia el duelo. Cabe aclarar que el duelo en este proyecto no se refiere solamente a la pérdida de un ser querido, es importante tener en cuenta que este proceso puede ser para cualquier tipo de pérdida, no relacionarlo siempre con la muerte. En este caso es con la imposibilidad del estudiante de asistir a clases. (Feijoo y Pardo, 2003, p.71)

Por otro lado, como resultado de la entrevista semi estructurada (Fontana, 2000) realizada a una docente especializada en aulas hospitalarias en Bogotá, surge la oportunidad de trabajar con un educando hospitalario con el que se desarrollaron procesos educativos en el área de inglés. Dichos procesos serán inclinados por el aprendizaje experimental, el cual es definido por Kolb (2015) como "el proceso por el cual el conocimiento es creado a través de la transformación de la experiencia... donde el conocimiento resulta de la combinación de la comprensión y transformación de la experiencia". (p.41). Es decir, el aprendizaje se basa en el proceso mismo de la vivencia, en el que a través de la práctica en contexto se busca crear la conceptualización que solidifique el aprendizaje y a su vez sea referencia en adelante. Se encuentra apropiado este enfoque ya que la creación del héroe permite que la experiencia sea reflejada en los resultados.

\section{OBJETIVO}

Teniendo en cuenta lo anterior, los objetivos guiarán el proyecto para llegar a los resultados 
esperados por medio de esta investigación. Como objetivo general se propone describir el manejo del duelo de un educando hospitalario a través de la creación de un héroe y la enseñanza del inglés. Así mismo se establecen los objetivos específicos en los que se pretende: incluir al educando hospitalario en procesos educativos y analizar el rol del inglés por medio de la creación en el proceso de duelo.

\section{POBLACIÓN Y PROCESO}

En esta sección se presentan los estudios previos referentes a la superación del duelo en aulas hospitalarias a través del aprendizaje del inglés con pacientes diagnosticados con enfermedades terminales o que requieren de una larga hospitalización. Esto se presenta dentro de los constructos establecidos, en los que la teoría existente nos permitió acercarnos a esta población. Los diferentes constructos se desarrollan desde los temas que son de interés para este proyecto; además, los autores enriquecen la reflexión y a la descripción del proceso inclusivo educativo de los educandos hospitalarios.

\section{DUELO}

De acuerdo con Feijoo y Pardo (2003) "El duelo es un mecanismo normal y saludable que sirve para asimilar una pérdida y recuperar las ganas de vivir... Aquí no nos referimos solo a la muerte, el duelo se experimenta también, frente a otras situaciones de pérdida, divorcio, desempleo, cambio de ciudad, etc." (p.71) Es así que tendremos que definir el duelo como un proceso de pérdida en el cual la persona, en este caso el estudiante, sufre por algo que ya no tiene, por una persona que ya no lo acompaña o un lugar que dejará de habitar. Es así como resulta fundamental el hecho de transitar el duelo de manera guiada y consciente. Específicamente desde el campo pedagógico se puede abordar el duelo teniendo en cuenta diferentes estrategias que permitan una transición lo más fluida posible con fin de superar cada etapa propuesta.

Para lo anterior contamos con un modelo basado en Kubler-Ross (2005), quien basa su estudio en cinco etapas durante el proceso del duelo, las cuales son: negación, ira, depresión, negociación y aceptación. A partir de esto se puede trabajar con las diferentes etapas a través de estrategias de arteterapia donde el educando hospitalario por medio de la pedagogía hospitalaria puede continuar con su interacción académica y social; esto aporta a la reducción del impacto y manejo del duelo desde el área pedagógica.

Aportando a la definición de duelo, Bowlby (1960) lo define como "todos aquellos procesos psicológicos, conscientes e inconscientes, que la pérdida de una persona amada pone en marcha, cualquiera que sea el resultado". Aunque la definición se refiere directamente a la pérdida en relación a la muerte, esto también está relacionado con el concepto de pérdida en sus distintos ámbitos y los procesos mentales que involucran el duelo y su control.

Lo que se observa en las definiciones de los diferentes autores es que el duelo es un proceso que afecta directamente a la persona ya que en su mayoría de casos se involucra la depresión, tristeza entre otras características que hacen al ser humano tener un problema, de allí que el acompañamiento psicológico juega un papel fundamental y más que todo en edades tempranas. Teniendo en cuenta ello, en este estudio el papel de la creación del héroe hace que el educando hospitalario muestre sus sentimientos, emociones y demás experiencias a través de un personaje en el cual se va a sentir identificado.

\section{ARTETERAPIA}

La creación de un héroe por medio del inglés como herramienta de gestión del duelo tiene 
una base en las necesidades de los alumnos de expresar su sentimiento de pérdida, esto ocurre luego de que se ven obligados a abandonar la escuela por su condición médica. Es ahí donde se involucra la arteterapia como instrumento para crear el héroe y donde consigue su validez; según Romero, (2014) "Aunque la arteterapia tiene un impacto en el campo de la expresión artística, su práctica se centra en las áreas de salud o servicios sociales. Los primeros arteterapeutas fueron artistas o profesores de arte interesados en los aspectos psicológicos y sociales de las personas." (p.2) Teniendo en cuenta esto, es la razón por la que este proyecto se centra en esta estrategia a través de la creación de un héroe en inglés, ya que los educandos hospitalarios tienen la posibilidad de expresar sus pensamientos, percepciones y la gestión del duelo. Asimismo, Klein (2006) apunta que "la arteterapia es un acompañamiento de personas en dificultad (psicológica, física, social o existencial) a través de sus producciones 245 artísticas, obras plásticas, sonoras, teatrales, literarias, físicas y de danza". (p.13). Como lo menciona el autor anteriormente la arteterapia es una forma donde cierta población puede expresar sus dificultades por medio del arte y la creación es por ello que, a través de este proceso, el enfoque experiencial es necesario debido a que es la base de su creación, teniendo en cuenta que el educando hospitalario puede recrear su realidad y de este modo expresar sentimientos y emociones que está atravesando.

Ahora, abordando el tema de la arteterapia como herramienta, Salazar (2019) afirma que "la arteterapia ha aportado interesantes herramientas de intervención para trabajar con niños que han vivido eventos traumáticos. En sí, la intervención desde la arteterapia genera una mejor comprensión de las emociones que los niños transmiten a través de las formas de comunicación artística que trabajan." (p.15) Como la autora mencionó anteriormente, es muy importante para los estudiantes, en este caso, teniendo en cuenta su limitación, encontrar una forma en la que puedan expresarse y demostrar los sentimientos que tienen en una determinada etapa de duelo o evento traumático, en este proyecto es el uso de un héroe, ya que el educando hospitalario es el personaje principal. El educando hospitalario puede jugar con su propia percepción y contexto.

Duncan (2007) concuerda con Salazar y los beneficios de la arteterapia al manifestar que "el trabajo con las emociones a través de la arteterapia mejora la calidad de las relaciones humanas porque se centra en el factor emocional, esencial en todo ser humano, ayudándonos a ser más conscientes de los aspectos oscuros, y facilitando así el desarrollo de la persona" (p.42). Para los educandos hospitalarios esta estrategia es atractiva y a través de la creación de héroes pueden trabajar en sus sentimientos de acuerdo a sus experiencias en este contexto, también cómo pueden manejar el duelo. Hay una parte fundamental que es el papel del profesor, quien da las instrucciones para que el duelo pueda ser gestionado y se pueda superar esta etapa en el menor tiempo posible teniendo poco impacto negativo en el estudiante.

A continuación, es necesario enfocarse en el papel del educando en el proceso creativo es por ello que para Klein (2006), "El trabajo de ponerse en forma recreativa no se hace en primera persona, sino en el artificio de describir un personaje que a veces permite que se revele con más autenticidad y profundidad, y esto es particularmente cuando el sujeto está demasiado cómodo y relajado en el lenguaje pseudointrospectivo". (p.17) A partir de la descripción realizada por este autor, la posibilidad que ofrecemos al alumno afectado por el abandono escolar es la de crear un héroe a través de este proceso, que le acompañe durante su duelo y le proporcione herramientas para superarlo. En este sentido, observaremos el proceso que tendrá el educando hospitalario durante su control y 
superación del duelo cuando deba abandonar la escuela. Sin embargo, es importante aclarar que no se va a realizar un proceso médico, sino una intervención pedagógica.

Continuando, Bassols (2006) afirma que "la arteterapia es un acompañamiento, un tratamiento, no es hacer un diagnóstico, por lo que el profesor acompañante debe promover el uso de herramientas artísticas para controlar el duelo, a partir de las necesidades, intereses y gustos del alumno." (p.19). Así como es mencionado por el autor, el profesor tiene el papel de acompañante, además de implementar la pedagogía hospitalaria es por ello que es importante destacar el papel que juega el profesor en esta etapa porque es quien puede diferenciar el proceso de cada alumno y qué tipo de proceso necesita desarrollar con el "educando hospitalario".

\section{EDUCANDO HOSPITALARIO}

El término educando hospitalario se refiere a niñas, niños o adolescentes con capacidades diversas, que por su condición de salud se pueden encontrar en ambientes educativos o en ambientes hospitalarios, ya que por su condición de salud habitan de manera temporal ambientes hospitalarios, estando ellos escolarizados o no y que requieren de una educación formal o no formal. (Florez, 2012 p.45) El participante de este proyecto es un educando hospitalario quien cursa tercero de primario y que por su condición de fibrosis quística se le imposibilita asistir a la escuela de forma regular y de cumplir con su plan académico.

Por un lado se debe considerar el cumplimiento de los derechos del educando hospitalario, ya que la educación es uno de los derechos básicos según la Constitución Política de Colombia (1991) en su capítulo 2, artículo 67 , manifiesta que "La educación es un derecho de la persona y un servicio público que tiene una función social: con ella se busca el acceso al conocimiento, a la ciencia, a la técnica, y a los demás bienes y valores de la cultura", es por ello que se debe brindar un proceso educativo de calidad a lo largo del tratamiento sin excluirlo, ni por su situación de salud, ni por el lugar en el que vive; además, teniendo en cuenta su estado psicológico y físico, que son claves para conseguir un estado de salud ideal (Castro, 2015 p.22). Gracias a la estrategia utilizada en este proyecto se puede brindar al educando hospitalario la oportunidad de aprendizaje en inglés en procesos de arteterapia como lo es en la creación del héroe y el manejo del duelo.

Por otro lado, es importante tener en cuenta el contexto donde reside el educando hospitalario, este puede ser tanto un hospital como en el hogar, dependiendo de la gravedad de la enfermedad y también de la intensidad del tratamiento, y el proceso educativo que este está llevando a cabo, esto es definido como pedagogía hospitalaria (Castro, 2019 p.66). Es por ello que este proyecto va enfocado a un educando hospitalario quien se encuentra en hospitalización domiciliaria además que está llevando su proceso académico en su residencia, teniendo en cuenta que este no se cumple a cabalidad puesto que la pedagogía hospitalaria no es implementada, es por ello que es necesario un proceso inclusivo teniendo en cuenta las necesidades del educando.

La pedagogía hospitalaria también desafía la pérdida del contexto académico, la importancia de los logros que el educando hospitalario alcanza a lo largo del proceso, y su impacto en los estados psicológicos y físicos, también acorta las distancias entre la escuela, el hospital y la familia, disminuyendo drásticamente las posibilidades de sufrir síndrome de hospitalización. (González, 2019 p.20) Como lo autor lo manifiesta, es importante que el educando tenga ciertos logros los cuales deben 
ser realistas, para que así mismo su proceso sea llevadero y disminuya las probabilidades de sufrir ciertos traumas o síndromes, además de sobrellevar el duelo. Es allí donde desde el quehacer pedagógico se aborda el manejo del duelo a través de diferentes estrategias que facilitan el proceso, este proyecto va enfocado hacia una nueva perspectiva que permite al educando continuar con su proceso académico en permanente contacto con su contexto previo.

Los niños con dificultades de salud o los adolescentes no pueden entender el hospital sólo como un lugar de dolor y sufrimiento. Según Salgado (2020), "los educandos hospitalarios están en sus habitaciones mientras no están sometidos a procedimientos médicos, consultaso exámenes (durante el período de hospitalización) o en el proceso de hospitalización en casa, ya que estos espacios son propicios para continuar sus estudios. (p.101) y es necesario que siempre encuentren allí un ambiente que pueda ser utilizado para el juego y el aprendizaje a través de actividades pedagógicas y recreativas. La literatura señala que en la hospitalización infantil, la principal fuente de estímulo para que los niños se desarrollen es la persona que todos los días los involucra en su cuidado, ya sea para las comidas o la higiene, o el juego, o los procedimientos técnicos de enfermería. (Salgado, 2020 p.102) La implicación personal de quien cuida transmite al niño enfermo una experiencia esencial, el contacto humano. Partiendo de este entendimiento, la prestación de cuidados sanitarios al niño necesita cubrir sus necesidades sanitarias ampliadas, ya sean: emocionales, sociales, familiares, culturales o ambientales. Así, se vuelve fundamental incluir una propuesta de atención que pueda comprender acciones pedagógicas durante el período de hospitalización.

Por ello, en el periodo de hospitalización es necesario de una disciplina pedagogíahospitalaria cuyo manual de funciones tiene como objetivo la intervención de la acción desde el aprendizaje y la enseñanza para el desarrollo y el crecimiento de los educandos hospitalarios, de los educandos domiciliarios y de los habitantes temporales de los entornos hospitalarios. Es necesario pensar en su existencia para abordar la construcción de conocimientos sobre aspectos espirituales, psicológicos-sociales. Por otro lado, modificar la estructura de aprendizaje y el conocimiento para vivir desde la diversidad. En un contexto específico por la ley de la naturaleza, como condición de vida acorde a la humanidad y a favor de la sociedad. (Flórez, 2012) Las aulas hospitalarias manejan estrategias totalmente diferentes a las que se utilizan en las aulas regulares, así como las relaciones profesoralumno. En las aulas regulares, los profesores dependen de la asistencia de los alumnos y en caso de alguna irregularidad en esta materia, es el profesor el encargado de pedir las razones del alumno; en el aula hospitalaria, el educando hospitalario siempre estará esperando la llegada del profesor, ya que son ellos los que permanecen permanentemente en el contexto.

\section{METODOLOGÍA}

Este proyecto usa el método cualitativo como medio de estudio para tener una visión en el desarrollo de un proceso educativo en un aula no tradicional con una población como los educandos hospitalarios. Para esto, Christiensen (2014) afirma que "en el método cualitativo, diferentes grupos construyen sus realidades 0 perspectivas, y estas construcciones sociales, influyen en cómo ellos ven o entienden sus mundos, lo que ven como normal o anormal y cómo deberían actuar" (p.85). Este método es preciso para el presente estudio ya que a través de las sesiones se tiene en cuenta las experiencias del educando hospitalario, así como sus opiniones, motivaciones y actitudes en su contexto, estas se tienen el cuenta con 
el objetivo de que su proceso educativo sea inclusivo y se entienda su proceso de duelo a través de ellas.

Siguiendo la línea del método antes mencionado, la siguiente sección nos da a conocer el enfoque usado en el proyecto, el cual ayuda a centrar los análisis presentados en las sesiones.

El enfoque usado para el presente estudio es el estudio de caso, Christiensen (2014) lo define como un estudio que involucra un caso específico con el propósito de entender un proceso más general basado en el análisis de un caso individual (p. 582). Este enfoque permite obtener más información sobre la situación del educando hospitalario y su contexto educativo, así para entender su proceso de duelo.

\section{CONTEXTO}

El contexto hospitalario brinda la posibilidad de desarrollar este proyecto, lo cual permite analizar el proceso educativo del educando hospitalario y su inclusión en este campo. Así mismo, se involucra en el proceso, personas con las que el educando hospitalario convive diariamente.

\section{PARTICIPANTE}

El educando hospitalario es un niño de 7 años, quien en el tiempo que se realizó la investigación está cursando tercer grado y por su diagnóstico se le imposibilita asistir al aula regular. Guillén, (2002) establece que el educando hospitalario puede verse afectado por su alejamiento de su contexto. Es un niño con gusto hacia los superhéroes además del interés que muestra por aprender el inglés, factores que facilitan el proceso de la creación del héroe.

En adición, el estudiante vive con su mamá quien es la responsable y quien lo autorizó para participar en este estudio; también vive con su hermana, y debido a su condición está en constante acompañamiento de profesionales. Dado su diagnóstico se presenta un impedimento para realizar actividades físicas de alta demanda.

\section{MUESTRA}

El término Critical Sampling (Creswell, 2012, p. 208) nos permite entender el fenómeno de educando hospitalario, teniendo en cuenta experiencias reflejadas a lo largo de la creación del héroe. La labor de los docentes investigadores dentro de este proceso es guiar al educando hospitalario a desarrollar las diferentes actividades de acuerdo a su experiencia y contexto. Como investigadores se nos permite dar las herramientas para crear un ambiente enriquecedor.

\section{INSTRUMENTOS $Y$ MÉTODOS DE RECOLECCIÓN DE DATOS}

En relación con el objetivo de este proyecto, se hacen necesarios ciertos instrumentos de recolección de datos los cuales son apropiados para analizar. Partiendo de ello, se seleccionaron tres instrumentos:

- $\quad$ Entrevistas: Se utilizó este instrumento ya que "demuestra las ideas de los entrevistados sobre el fenómeno de interés, también, soporta los pensamientos y creencias de una comunidad que es parte del estudio" (Christensen 2014). La entrevista permitió observar aspectos inesperados, los cuales son importantes en el estudio, esta fue desarrollada en una conversación natural para que no hubiera presión sobre el educando hospitalario, esta fue aplicada al inicio del proceso para tener una claridad del contexto y la vida del participante.

- $\quad$ Artefactos del estudiante: los artefactos cumplen un rol necesario en todo el proceso, ya que por medio de ellos se analizaron aspectos importantes en el proceso, estos son representados por la creación del héroe. Christensen (2014), define este instrumento 
como un material original que permite al estudiante entender eventos históricos, así como experiencias. Este instrumento determina cómo es la visión del estudiante en su manejo del duelo a través de la creación del héroe en inglés

- Notas post-facto: Este instrumento fue escogido, debido a que durante las sesiones se enfocó realizar las actividades y no se tenía la oportunidad de tomar las notas en el momento, por ello es necesario escribirlas después de cada sesión desarrollada, ya que no sería apropiado "registrar en escrito mientras ocurre el proceso", como lo expresa Lankshear \& Knobel (2004, p.229). Después de desarrollada la sesión las notas post-facto fueron escritas teniendo en cuenta la mayoría de detalles percibidos con el objetivo de analizarlos y reflexionar sobre los objetivos del presente estudio.

\section{SESIONES}

El grupo de sesiones de creación de héroe en inglés fueron desarrolladas del mes de mayo a junio del 2021. Cada sesión fue basada en el enfoque trabajado y su estrategia. El enfoque es el aprendizaje por medio de la experiencia (Kolb 2015 p.41) el cual permite que el educando hospitalario involucre su experiencia en el proceso de aprendizaje en este proyecto en la creación del héroe siendo él el protagonista para el manejo del duelo. Así mismo incluye su contexto (familia, amigos, escuela, proyecto de vida, hospital) por medio de la arteterapia (Romero, 2014 p.2), estrategia que fue usada para la creación del héroe.

\section{INTERPRETACIÓN}

En esta sección se encuentra la interpretación de los resultados de la investigación, en el cual se usa el método de teoría fundamentada la cual es definida como una metodología que captura la diversidad de hechos, datos e información además de experiencias de la realidad (Behrens \& Prigol, tomado de Charmaz 1983 p.3). Esto permite que el docente investigador pueda familiarizarse con el problema y trabajar directamente con el fenómeno para mejorar ideas y así hacer una investigación más completa. Por esta razón este método cumple con las características que se adaptan a los objetivos de esta investigación.

De acuerdo a lo anterior, se han establecido dos categorías la primera de ellas los procesos socioacadémicos donde se presentan ciertos factores que permiten mostrar el entorno y proceso académico sobre el educando hospitalario y la segunda las situaciones fluctuantes que se presentan a lo largo del estudio. Estas categorías reflejan características valiosas del educando hospitalario las cuales permitieron hacer reflexión sobre el proceso usando la estrategia de arteterapia y también aquellas características que se puede encontrar en el aula hospitalaria.

\section{PROCESOS SOCIO-ACADÉMICOS}

De acuerdo a los procesos sociales y académicos los cuales se llevan a cabo con un educando hospitalario, han de tenerse en cuenta diferentes características para que la educación brindada en el aula hospitalaria sea la apropiada para garantizar el derecho a la educación, es por ello que se debe tener en consideración las necesidades y el contexto.

La creación del héroe fue un facilitador del proceso, ya que era uno de los incentivos para involucrar al educando hospitalario, además de converger sus contextos familiar, social, hospitalario, y escolar siendo estos los principales factores influenciadores en el manejo del duelo. Este proceso está articulado por el aprendizaje del inglés siendo este el enfoque académico. 
Se debe tener en cuenta que el educando hospitalario quien fue la población en la que se enfocó este estudio es variable, con ello se quiere decir que para él hay una gran variedad de factores que dificultan un proceso académico constante. Un ejemplo de ello es que debido a sus tratamientos médicos deben detener su proceso de aprendizaje. Debido a ello, es importante que cualquier tiempo que se tenga con el educando hospitalario sea aprovechable.

Durante las sesiones realizadas, se logra evidenciar la relación del educando con su contexto social y familiar, siendo este último con el que más sostiene interacciones significativas, un factor facilitador en el manejo del duelo, ya que da un soporte afectivo al educando hospitalario. Adicionalmente, se hace presente la necesidad de un acompañamiento médico, dado que teniendo en cuenta el diagnóstico, se puede realizar un tratamiento en hospitalización domiciliaria.

Más allá del diagnóstico de corta expectativa de vida, el educando manifiesta deseos a futuro (muchos de ellos inmediatos), tales como el aprendizaje del inglés, el retomar espacios académicos con sus amigos, el disfrutar momentos específicos en familia. Otros deseos por el contrario a mediano y largo plazo, como, sus logros académicos, su incursión en nuevas actividades deportivas, esto se dio a conocer gracias a la proyección del héroe.

\section{SITUACIONES FLUCTUANTES}

En esta categoría, se muestran aquellas situaciones sobre el educando hospitalario, el proceso pedagógico y el entorno las cuales fueron inesperadas, pero aportan un gran valor ya que se puede entender ciertos aspectos fluctuantes que afectan el manejo del duelo del participante. El manejo de estas situaciones afrontadas fue un desafío, puesto que se debe cambiar el curso de las actividades y centrarse en la situación, hecho que permite reflexionar sobre el proceso académico y las variantes de este.

De acuerdo a las situaciones que se vivieron, la primera de ellas presentada fue el espacio el cual el educando hospitalario tenía destinado para desarrollar sus sesiones, esté era cambiante en tanto no hubo un lugar definido para la totalidad del proceso, dicho espacio dependía de circunstancias familiares, médicas o de transporte, lo que causaba en el educando hospitalario cambios abruptos, que una vez más resaltaban la importancia de adaptarse como educador a sus necesidades y contextos.

Por un lado, la participación de la familia genera inestabilidad en el proceso académico del estudiante, al cambiar sus espacios innegablemente cambiaban las personas que lo acompañaban, y estas a su vez generaban diferentes emociones en el educando hospitalario que se veían reflejadas en su comportamiento y rendimiento académico, es así como la presencia de familiares de mayor edad propiciaba en el educando hospitalario una mayor dependencia y asistencialidad en el momento de desarrollar las tareas, mientras que ante la presencia de pares el educando hospitalario mostraba mayor independencia y liderazgo.

Por último, enfocándonos en la actitud y personalidad del educando, las sesiones varían de acuerdo a su estado emocional, el cual cambia de acuerdo a variables mencionadas anteriormente como lo son; familiares y médicas, que indudablemente afectan sus emociones en cuanto exhortan al educando a tomar posición frente a su contexto inmediato, pese a esto el educando siempre estuvo en disposición de tomar las sesiones y enfrentar los retos que estas conllevan. 
Teniendo en cuenta lo anterior, se recopiló la reflexión y las experiencias a lo largo de la investigación con el fin de concluir como desde el campo pedagógico se da el manejo del duelo a través de la creación del héroe y la enseñanza del inglés para un educando hospitalario.

\section{CONCLUSIONES}

A lo largo de la investigación, resulta imperante la necesidad de reflexionar sobre el rol docente y sus campos de acción, y como desde el mismo se pueden abordar poblaciones que suelen ser olvidadas o cuyas necesidades educativas se ven forzosamente pospuestas por una gran variedad de circunstancias. Circunstancias que pueden ser abordadas con la ayuda de enfoques pedagógicos y artísticos, que articulados brindan la posibilidad de romper barreras y retomar procesos académicos.

En tal sentido, la investigación aborda el manejo del duelo en la pedagogía hospitalaria a través de la creación de un héroe y la enseñanza del inglés, de las evidencias anteriormente descritas es vital destacar la importancia de desarrollar dichos procesos académicos en esta población, más allá de la disciplina impartida o las técnicas utilizadas, el manejo del duelo en educandos hospitalarios resulta vital para su continuidad académica, al mismo tiempo puede significar mejoras en sus estados de ánimo, así como puede ser un agente unificador en entornos familiares.

Es así cómo se incorpora al educando hospitalario a la experiencia más cercana a su previa regularidad escolar, llevando dicho ambiente a través de experiencias a contextos inusuales como el hogar o el hospital, lo que no solo garantiza el derecho a la educación del educando hospitalario, sino que también hace un aporte a su proyecto de vida, estableciendo objetivos a corto, mediano y largo plazo que incentiven al educando a no abandonar la escuela y su formación ya sea ésta regular o no.

Es por ello, y con el fin de sobrellevar posibles síndromes, traumas o secuelas que deje la experiencia del duelo, resulta necesaria la participación de otros profesionales que desde sus campos de acción estén en capacidad de dar un manejo adecuado a las emociones a mediano y largo plazo, y que, por consiguiente, aporten a cerrar los ciclos necesarios en el manejo del duelo.

En consecuencia, esta investigación apunta a explorar nuevos contextos en los cuales, a través de la pedagogía, se puedan abordar poblaciones usualmente no consideradas prioritarias académicamente, y que generan un gran impacto en el ser y el quehacer docente. Además de que se deberían implementar diferentes políticas educativas las cuales aporten a los contextos y poblaciones que no se encuentran en el aula regular.

\section{REFERENCIAS BIBLIOGRÁFICAS}

Barbero, J. C. G. M. (2007). Arteterapia, Parálisis Cerebral y Resiliencia. ArteterapiaPapeles de arteterapia y educación artística para la inclusión social, 2, 169179.

Bassols, M. (2006). La arteterapia, un acompañamiento en la creación y la transformación. Arteterapia. Papeles de arteterapia y educación artística para la inclusión social, 1, 19-25.

Behrens, M, \& Prigol, E. (2019). Teoría Fundamentada: metodologia aplicada na pesquisa em educação. Educação \& Realidade, 44(3), e84611. Epub 
Blanco, R (2006). La inclusión en educación: una cuestión de justicia y de igualdad. Sinéctica, Revista Electrónica de Educación, (29),19-27. [fecha de Consulta 5 de Abril de 2021]. ISSN: 1665-109X. Disponible en: https://www.redalyc.org/articulo. oa?id=99815739003

Bowlby, J. ( 1960b). " "Grief and mourning in infancy and early childhood".' Psychoanal. Study Child, 15, 9-52.

Carbo, L. (2016) Arteterapia e inteligencia emocional: análisis de las cualidades emocionales a través del proceso creativo en Arteterapia (Tesis de maestría) Universidad del desarrollo. Recuperado de: http://repositorio. udd.cl/bitstream/handle/11447/1533/ Arteterapia

$\% 20$ e $\% 20$ inteligencia $\% 2$ 0emocional. pdf?sequence $=1$ \&isAllowed $=y$

Castro, M \& Santana, D (2015). Diseño e Implementación de una Estrategia Didáctica que Oriente la Práctica en contextos de salud y hospitalarios para las Estudiantes de IX Semestre de Licenciatura en Pedagogía Infantil de la Fundación Universitaria los Libertadores. Bogota, Colombia.

Castro, C. y Salgado, C. (2019). Experiencias de aprendizaje en el aula hospitalaria. En Maneras de sentir las pedagogías hospitalarias y domiciliarias (pp. 63-69). Universidad Nacional de Colombia

Christiensen, L\& Jhonson, B. (2014). Educational Research. Fifth edition. University of south of Alabama. SAGE Publications. ISBN 978-1-4522-4440-2
Creswell J. (2012). Educational Research. Planning, Conducting, and Evaluating Quantitative and Qualitative Research. Pearson Education. University of Nebraska-Lincoln. ISBN-10 0-13136739-0

Duncan, N. (2007). Trabajar con las Emociones en Arteterapia. Arteterapia. Papeles de arteterapia y educación artística para la inclusión social, 2, 39-49.

Feijoo Portero, P., \& Pardo Porto, A. B. (2017). Muerte y educación. Tarbiya, Revista De Investigación E Innovación Educativa, (33).

Flórez, L. (2012). Pedagogía de la Salud Efectos de la dimensión psicológica del aprendizaje lingüístico sobre el aprendizaje psicomotor en educandos pediátricos domiciliarios. Universidad de la sabana. Bogotá, Colombia

Fontana, F., \& Frey, J. (2000). The interview: From structured questions to negotiated text. In N. K. Denzin \& Y. S. Lincoln (Eds.), Handbook of qualitative research (pp.645-672) Thousand Oaks, CA: Sage

Gobierno de Colombia. (1991). Constitución Política de Colombia. Capítulo 2, artículo 67. Bogotá

González, A. O. (2019). Entrevista con Olga Lizasoáin Rumeu sobre pedagogía hospitalaria: trayectorias de desarrollo intelectual, conquistas profesionales y desafíos de futuro

Guillén C, (2002). Actuaciones educativas en aulas hospitalarias: atención escolar a niños enfermos. Narcea. Madrid. 
Klein, J. P. (2006). La creación como proceso de transformación. Arteterapia. Papeles de arteterapia y educación artística para la inclusión social, 1, 11-18.

Kolb, D. (2015). Experiential Learning: Experience as the Source of Learning and

Development. Second Edition. New Jersey. United States of America. Pearson

Education, Inc. ISBN - 10: 0-13-389240-9.

Kübler-Ross, E., \& Kessler, D. (2005). On grief and grieving: Finding the meaning of grief through the five stages of loss. Simon and Schuster.

Lankshear C. \& Knobel M. (2004) A Handbook for Teacher Research: form design to implementation. Open University Press. England. Berkshire. ISBN 0335210643

Romero, B. L. (2004). Arte terapia. Otra forma de curar. Educación y futuro: revista de investigación aplicada y experiencias educativas, (10), 101-110.

https://www.revistas.ufg.br/fen/article/view/827

Salgado, C. (2020). Miradas de la pedagogía hospitalaria: reflexiones encarnadas de un docente hospitalario. Infancias Imágenes, 19(1), 100-107.

Salazar, E (2019). Arteterapia como medio psicoterapéutico para eventos traumáticos. Universidad Santo Tomás, Bogotá, Colombia

Steinke, S. M., Elam, M., Irwin, M. K., Sexton, K., \& McGraw, A. (2016). Pediatric Hospital School Programming: An Examination of Educational Services for Students who are Hospitalized. Research, Advocacy, and Practice for Complex and Chronic
Conditions, 35(1), 28-45. https://doi. org/10.14434/pders.v35i1.20896

UNESCO (2015). Guidelines for inclusion: ensuring access to education for all. Paris, Francia. Retrieved from https:// unesdoc.unesco.org/ark:/48223/ pf0000140224 